Supplementary Material

\title{
Subaerial speleothems and deep karst in central Sweden linked
}

\author{
to Hirnantian glaciations $-{ }^{87} \mathrm{Sr} /{ }^{86} \mathrm{Sr}$ isotope ratio of selected
}

brachiopod shells, and results of Energy-dispersive-X-ray

spectroscopy

Björn Kröger ${ }^{1}$, Jan Ove R. Ebbestad ${ }^{2}$, Oliver Lehnert ${ }^{3,4}$, Clemens V. Ullman ${ }^{4,6}$, Christoph Korte $^{5}$, Robert Frei ${ }^{5,7}$, and Christian M. Ø. Rasmussen ${ }^{4,8}$

${ }^{1}$ Finnish Museum of Natural History, P.O.Box 44 (Jyrängöntie 2), 00014 University of Helsinki, Finland; bjorn.kroger@helsinki.fi

${ }^{2}$ Museum of Evolution, Uppsala University, Sweden; jan-ove.ebbestad@em.uu.se

${ }^{3}$ GeoZentrum Nordbayern, Lithosphere Dynamics, University of Erlangen-Nürnberg,

Schlossgarten 5, D-91054 Erlangen, Germany; oliver.lehnert@fau.de.

${ }^{4}$ Department of Geology, Lund University, Lund, 223 62, Sweden.

${ }^{5}$ Department of Geosciences and Natural Resource Management, University of Copenhagen,

Øster Voldgade 10, 1350 Copenhagen K, Denmark; cu@ign.ku.dk; korte@ign.ku.dk;

robertf@ign.ku.dk

50partment of Geology, Lund University, Lund, 223 62, Sweden; oliver.lehnert@@geol.lu.se, christian.rasmussen@geol.lu.se

${ }^{6}$ University of Exeter, Camborne School of Mines, Penryn Campus, Treliever Road, Penryn,

Cornwall,TR109FE,UK; c.ullmann@exeter.ac.uk 
${ }^{7}$ Nordic Center for Earth Evolution (NordCEE), University of Copenhagen.

${ }^{8}$ Natural History Museum of Denmark, University of Copenhagen, Oster Voldgade 5-7, 1350

Copenhagen K; christian@snm.ku.dk

\section{1. ${ }^{87} \mathrm{Sr} /{ }^{86} \mathrm{Sr}$ isotope ratio of selected brachiopod shells}

The stratigraphic position, additionally, was tested with the ${ }^{87} \mathrm{Sr} /{ }^{86} \mathrm{Sr}$ isotope ratio of selected brachiopod shells (Table 1). The isotope ratios of marine shells of Hirnantian age should be between 0.707945-0.707868, slightly higher than Katian ratios, and lower than Silurian ratios

(LOWESS 5 skeleton dataset, McArthur et al., 2012). Our values, obtained from brachiopod shells from Hirnantian beds of Osmundsberget, Kallholn and Solberga, are consistently much higher than expected (Table 3). This is probably a result of early diagenetic overprint and influence of meteoric water (compare Brand, 1991).

\section{Results Energy-dispersive-X-ray spectroscopy (EDS) of the speleothems}

A semiquantitative EDS of a sample S1B (same see in main paper, Table 2) from the laminated, peloidal grainstone (stromatolite-like speleothem) of the Solberga quarry was taken. The sample was Au coated for scan microscopy. The result indicates the presence of low MgCalcite in the micrite of the speleothem (Fig. 1). 


\section{REFERENCES CITED}

Brand, U., 1991, Strontium isotope diagenesis of biogenic aragonite and low-Mg calcite:

Geochimica et Cosmochimica Acta v. 55, p. 505-513.

McArthur, J.M., Howarth, R.J., and Shields, G.A., 2012, Strontium Isotope Stratigraphy: The Geologic Time Scale 2012. Edited by Gradstein, F.M., Ogg, J.G., Schmitz, M., and Ogg, G., Elsevier, New York, p. 127-144. 
Table 1. List of results of ${ }^{87} \mathrm{Sr} /{ }^{86} \mathrm{Sr}$ ratios from brachiopod shells within the Boda Limestone (UB Mbr., Upper Boda Member; BC Mbr., Boda Core Member) and the overlying Glisstjärn Formation (Glisst. Fm).; Kallholn = Kallholn southern entrance wall of Suzuki et al. (2009), Osmundsberget localities = Osmundsberget 1, 4, 5 of Ebbestad and Högström (2007); Solberga south wall: N6058’57.3”, E015¹3’00.8”= locality Solberga 2 of Ebbestad and Högström (2007). Data was obtained using a Thermal Ionization Mass Spectrometer (TIMS).

\begin{tabular}{|c|c|c|c|c|}
\hline Sample & Taxon & Locality & ${ }^{87} \mathrm{Sr} /{ }^{86} \mathrm{Sr}$ ratio & Stratigraphy \\
\hline 10 & Hindella & Osmundsberget 1 & 0,707968 & $\begin{array}{l}\text { BC Mbr, } \\
\text { Katian }\end{array}$ \\
\hline 02 & Brevilamnulella kjerulfi? & Kallholn & 0,708847 & $\begin{array}{l}\text { UB Mbr, } \\
\text { Hirnantian }\end{array}$ \\
\hline 03 & Brevilamnulella kjerulfi & Kallholn & 0,708175 & $\begin{array}{l}\text { UB Mbr, } \\
\text { Hirnantian }\end{array}$ \\
\hline 04 & Clorilamnulella ? sp. & Kallholn & 0,708361 & $\begin{array}{l}\text { UB Mbr, } \\
\text { Hirnantian }\end{array}$ \\
\hline 07 & Brevilamnulella kjerulfi ? & Kallholn & 0,708168 & $\begin{array}{l}\text { UB Mbr, } \\
\text { Hirnantian }\end{array}$ \\
\hline 09 & Clorilamnulella osmundsbergensis & Osmundsberget 1 & 0,708199 & $\begin{array}{l}\text { UB Mbr, } \\
\text { Hirnantian }\end{array}$ \\
\hline 05 & Brevilamnulella kjerulfi & Osmundsberget 5 & 0,708416 & $\begin{array}{l}\text { UB Mbr, } \\
\text { Hirnantian }\end{array}$ \\
\hline 08 & Brevilamnulella kjerulfi & Osmundsberget 1 & 0,708466 & $\begin{array}{l}\text { UB Mbr, } \\
\text { Hirnantian }\end{array}$ \\
\hline 06 & Brevilamnulella umbosulcata & Solberga, south wall & 0,708080 & $\begin{array}{l}\text { UB Mbr, } \\
\text { Hirnantian }\end{array}$ \\
\hline
\end{tabular}




\begin{tabular}{|c|c|c|c|c|}
\hline Sample & Taxon & Locality & ${ }^{87} \mathrm{Sr} /{ }^{86} \mathrm{Sr}$ ratio & Stratigraphy \\
\hline 11 & Brevilamnulella umbosulcata & Solberga, south wall & 0,708027 & $\begin{array}{l}\text { UB Mbr, } \\
\text { Hirnantian }\end{array}$ \\
\hline 01 & Brachiopoda indet. & Solberga, south wall & 0,708556 & $\begin{array}{l}\text { fissure filling, } \\
\text { Silurian? marl }\end{array}$ \\
\hline 12 & Chlorilamnulella osmundsbergensis & Osmundsberget 1 & 0,708428 & $\begin{array}{l}\text { Glisst. Fm, } \\
\text { Hirnantian }\end{array}$ \\
\hline
\end{tabular}

Figure 1. Results of the EDS analysis of sample S1B from Solberga quarry, south wall, fissure crust. Note the elevated Au values, which are a result from the coating of the sample. 
Mineralogical laboratory, Department of Geosciences and Geography, University of Helsinki

\section{Sample:}

\begin{tabular}{|l|l|}
\hline Electron microprobe & Jeol Superprobe \\
\hline Model: & JXA-8600 \\
\hline Analysis type: & $\begin{array}{l}\text { EDS } \\
\text { semiquantitative }\end{array}$ \\
\hline Current: & $1 \mathrm{nA}$ \\
\hline Calibrated & March 2011 \\
\hline Accelerating voltage: & 15.0 \\
\hline Analysed: & $19.2 .201410: 54$ \\
\hline
\end{tabular}

\section{Au coated Calcite}
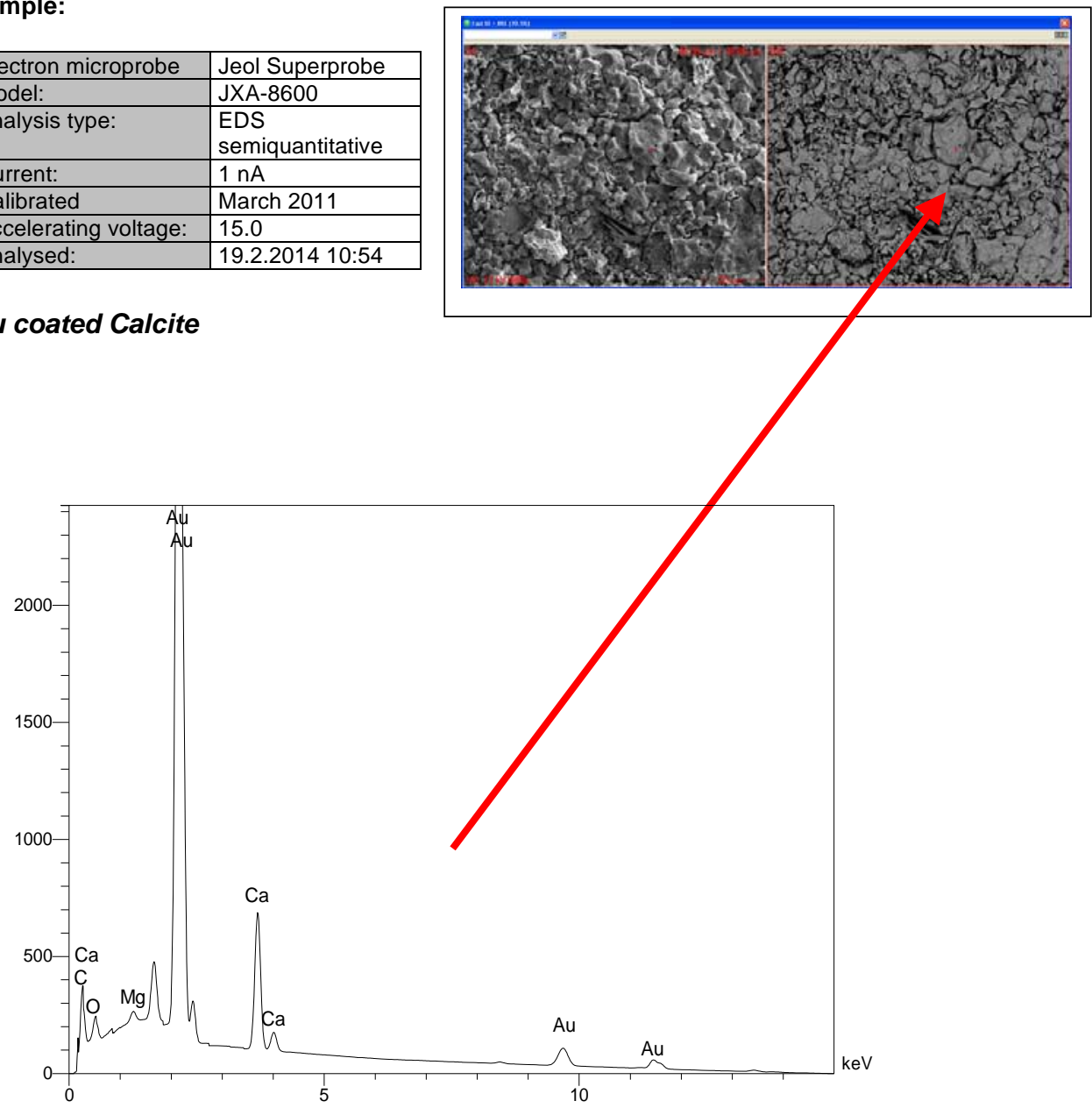

Quantitative Results

\begin{tabular}{lllllll}
\hline Elt & XRay & W\% & A\% & Formula & Ox\% & Cat\# \\
\hline C & Ka & 7.80 & 47.18 & & 0.00 & 0.00 \\
O & Ka & 2.34 & 10.64 & 0.00 & 0.00 \\
Mg & Ka & 0.24 & 0.72 & 0.00 & 0.00 \\
Ca & Ka & 5.81 & 10.53 & 0.00 & 0.00 \\
Au & La & 83.81 & 30.92 & 0.00 & 0.00 \\
& & 100.00 & 100.00 & 0.00 & 0.00 \\
\hline
\end{tabular}


